\title{
Rehabilitation Robotics: a Wearable Exo-Skeleton for Tremor Assessment and Suppression*
}

\author{
E. Rocon, A.F Ruiz, J.L. Pons, Member, IEEE \\ Instituto de Automática Industrial-CSIC \\ Ctra. Campo Real km. 0.200, 28500 Arganda del Rey, Spain \\ jose.pons@ieee.org
}

\author{
J.M. Belda-Lois, J.J. Sánchez-Lacuesta \\ Instituto de Biomecánica de Valencia \\ Universidad Politcnica de Valencia \\ Camino de Vera s/n., 46022 Valencia, Spain
}

\begin{abstract}
There is a need for wearable powered upper limb exoskeletons able to apply forces to the upper limb for use by people with disabilities and/or limb weakness or injury. The robotic exoskeleton called WOTAS (Wearable Orthosis for Tremor Assessment and Suppression) presented in this paper will provide a means of testing non-grounded control strategies in order to help these people. For instance, biomechanical loading, in particular, viscous loading of the upper limb has been proposed in the literature as a means for suppressing pathologic tremor. This article describes in detail the general concept for WOTAS, outlining the special features of the design and selection of system components.
\end{abstract}

Index Terms-Rehabilitation Robotics, HRI, tremor suppression, impedance control

\section{INTRODUCTION}

The scientific community is becoming more and more interested in the so-called Rehabilitation Robotics. One of the specific and important common aspects to the field of rehabilitation robotics is the intrinsic interaction between human and robot. This interaction has a twofold scenario, namely, first, a cognitive interaction by means of which the human is able to control the robot while the robot transmits feedback to the human; secondly, a biomechanical interaction leading to the application of controlled forces between both actors.

On the one hand, a typical example of the cognitive interaction is the one being developed through the EMG control of artificial robotics prostheses, [1]. Here, the human myoelectrical signals are used to develop control commands to drive an intelligent prosthesis. Force feedback can be implemented a number of means. On the other hand, a classical example of biomechanical interaction is found in exoskeleton based functional compensation of human gait. Here, the robotics exoskeleton, applies functional compensation by supporting human gait, i.e. by stabilizing the stance phase.

In general, rehabilitation robots can be classified, see [2], under three categories:

1) Posture support mechanisms

2) Rehabilitation mechanisms

3) Robots to assist or replace body functions

\footnotetext{
* The work presented in this paper has been carried out with the financial support from the Commission of the European Union, under contract $\mathrm{n}^{o}$. QKL6-CT-2002-00536, "DRIFTS - Dynamically Responsive Intervention for Tremor Suppression"
}

Tremor is characterized by involuntary oscillations of a part of the body. The most accepted definition is as follows: "an involuntary, approximately rhythmic, and roughly sinusoidal movement" [3]. Tremor is a disabling consequence of a number of neurological disorders. As a function of the particular kind of tremor, an uncontrolled oscillation of the upper limbs, trunk and even legs could occur. In addition, tremor can be present during rest (Parkinson Disease), while maintaining a posture (essential tremor) or during kinetic tasks [4]. It has been established in the literature that most of the different types of tremor respond to biomechanical loading. Hogan, [5], established that the human upper limb is impedance controlled during manipulation. In particular, it has been clinically tested that the shunt increase of damping and/or inertia in the upper limb leads to a reduction of the tremorous motion, i.e. the change in impedance characteristics of the upper limb has a direct effect on the tremor characteristics.

This phenomenon gives rise to the possibility of an orthotic management of tremor. An orthosis is a wearable device (exoskeleton) that acts in parallel to the affected limb. In the case of tremor management, the exoskeleton must apply a damping or inertial load to a selected set of limb articulations. As a wearable device, it must exhibit a number of aesthetics, cosmetic as well as functional characteristics.

In the framework of the DRIFTS project, [6], WOTAS (Wearable Orthosis for Tremor Assesment and Supression) was presented with two main objectives: monitoring and diagnosis, and validation of non-grounded tremor reduction strategies. In this article we present the development of such robotic platform in its aspects related to human robot interaction. In the next section, the biomechanics of the upper limb is studied. Next, a brief introduction of WOTAS' architecture will be given. This will be followed by the introduction of a biomechanical model that describes the kinematics and dynamics of the combination upper arm-WOTAS. Eventually, the conclusions and future work are given.

\section{BIOMECHANICS OF THE UPPER LIMB}

Cancelling tremor by mechanical loading the upper limb by robotic exoskeletons implies an inherent physical interaction between user and robot. There are two main concerns regarding the biomechanics of this interaction: 
1) The user's tolerance to pressure

2) Mechanical characterization of the soft tissue of the upper-limb

\section{A. Tolerance to pressure}

Pressure distribution is one of the main concerns related to robotic based orthotics devices. The most important function of any orthotic device is related to load transmission to the body bony structures through the soft tissues. Therefore, there are several factors in relation with pressure that have to be taken into account, namely, safety, pain and comfort.

There are two basic strategies to manage an external load: concentrate loads over a small region with high tolerance to pressures or distribute the load over an area as big as possible to reduce the pressure. Last strategy match the strategy for preventing pain or injuries, so it is commonly adopted as the right one, but comfort can differ substantially from this approach. For instance, Krooskop et al. [7] have shown that mattresses with uniform pressure distribution can cause restless. Goonetilleke show in its work that there should be a threshold in which it is better concentrate than distribute forces, [8]. The reason for that should be the Spatial Summation Theory that claims that as the area of contact increases the number of excited skin receptors also increases and subsequently the comfort perception is worst. Two main aspects have been analyzed:

1) User perception of pressure

2) pressure threshold for discomfort

In the current study we've applied the methodology suggested by Gonzalez et al [9] to find-out the Pressure Discomfort Threshold (PDT). In the afore mentioned study, the methodology was applied to foot pressure distribution. But, results from this study suggested that the methodology could be appropriate to analyze the ability to support pressure in the upper limb.

Pressure discomfort threshold has been studied in nine subjects, all of them affected by tremor at the upper limb. The average age was 53.5 years old (std. dev. 21). Measurement points have been located taking into account the common placement of load transmission elements of upper-limb tremor suppression orthoses. These points were chosen on the dorsal and palmar side, over muscles and over bony areas in order to have a good representation of the different areas of the forearm. We used dynamometry for measurements (Figure 1). The indentor was an aluminum cylindrical cap with a contact flat surface of 1.3 $\mathrm{cm}^{2}$ adapted to the dynamometer. The dynamometer was connected to a computer by means of a data acquisition card. Pressure was applied five times on each point. The sequence of pressure application was randomized previously, to avoid the learning effect of the patient in each point.

In the study carried out, no significant differences have been found in pressure discomfort threshold between the points analyzed, and therefore no significant differences were found in the pressure sensitivities. Subsequently, there

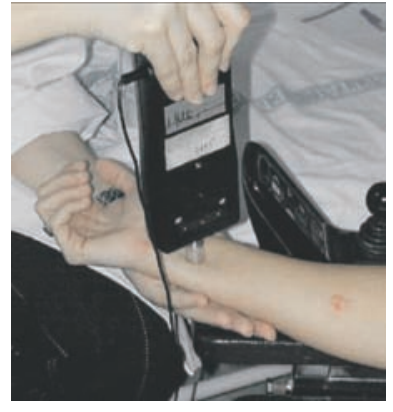

Fig. 1. Pressure sensitivity measurements.

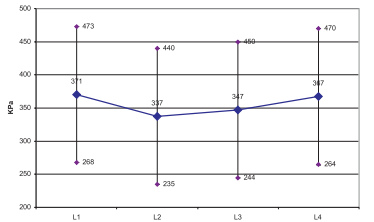

Fig. 2. Pressure tolerance threshold pattern. Measurement points versus tolerance to pressure

are no preferred locations to fix the exoskeleton force interface to the upper limb. Whatever point in the forearm can manage the same pressure in relation with comfort. Pressure thresholds pattern are shown in Figure 2.

The maximum pressure for discomfort found in the forearm was $230 \mathrm{kPa}$. In order to ensure comfort maximum values should have to be at least one order of magnitude under this figure.

\section{B. Mechanical characterization of soft tissues at the upper- limb}

Stiffness between the orthotic device and the body is a key factor to take into account in order to control a dynamic process such as tremor. The fixation of the orthotic device to the arm acts as a spring that can change significantly the behavior of the overall system. Soft tissues strain-stress curve is represented in Figure 3. As it can be seen, it is a curve with hysteresis. In our first approach, since the orthotic devices are attached once and then they have slow movements from this position, just the rising part of the stress curve was considered.

A study to characterize the soft tissues at different points of the upper limb has been performed. Measurement points have been located taking into account the common placement of load transmission elements of upper-limb orthoses. According to our study, no major differences related to gender could be found. It was also concluded

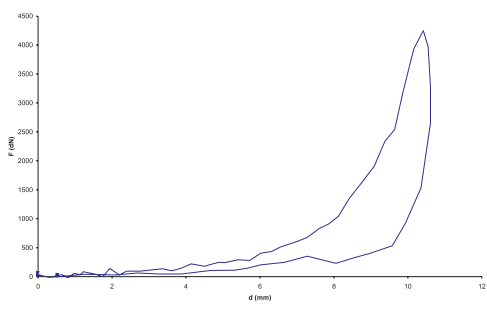

Fig. 3. Strain stress curve for the forearm. 


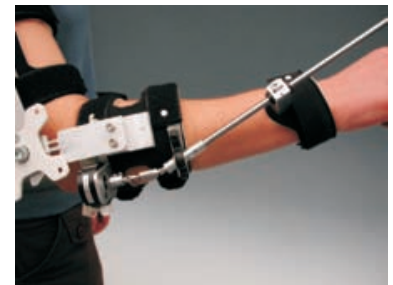

Fig. 4. View of the elbow and forearm joints in the WOTAS system.

that the soft tissues of the forearm could be modelled by a third order polynomial that describes the deformation of the tissue according to the force applied.

\section{WOTAS}

An orthosis is defined as a medical device that acts in parallel to a segment of the body in order to compensate some lost function. The upper limb main function is to guarantee that the hand implements its functions and reaches any point in the space, specially any point in human surface, in such a way, that the person can manipulate, draw on, and move objects, from or to the body. Therefore, the kinematic chain comprising shoulder, elbow, forearm, wrist, and hand, has a high mobility and prehension capacity with an infinite number of positions and functions. The upper limb is one of the most anatomic and physiologically complex part of the body.

The upper limb is very important because is able to execute cognitive, expression, and manipulation activities. Furthermore, it takes a crucial role when exploring the environment and in all reflex motor acts. Consequently, any alteration or pathology that affects the upper limb motion range, muscle power, sensibility, skin integrity, will alter its operation. The concept of WOTAS is to develop an active upper limb exoskeleton based on robotics technologies capable of applying forces to cancel tremor and retrieve kinematic information from the tremorous upper limb.

The WOTAS concept will provide a means of testing non-grounded tremor reduction strategies. This robotic device spans the elbow and wrist joints, being able to apply independent tremor suppression strategies to elbow flexo-extension, wrist flexo-extension and wrist pronosupination, see Figure 4.

\section{A. Mechanical design}

WOTAS is intended to suppress tremor in the three anatomical joints with higher contribution to tremor: Elbow flexion extension, Forearm pronation-supination, Wrist flexion extension.

WOTAS restricts the movement of wrist adductionabduction, nevertheless this movement is the less functional of all considered, [10]. Furthermore, as in the WOTAS concept, the fingers remain free and wrist abduction-adduction is dependent to the finger movements [11], the functional reduction is even lower.

The mechanical design of the joints for elbow flexionextension, and wrist flexion-extension are similar to other orthotic solutions and are based in the behavior of those physiological joints as hinges. The axis of rotation for

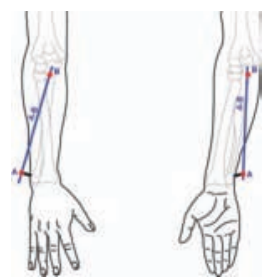

Fig. 5. Scheme of the pronation-supination control.

the elbow joint its placed in the line between the two epicondyles. The axis of rotation for the wrist joint is located in the line between the capital and lunate bones of the carpus.

The mechanical design for the control of the pronosupination movement is more complex and it is explained below.

1) Pronation-supination control: Pronation-supination is the rotational movement of the forearm along its longitudinal axis and involves two joints which are mechanically linked [11]:

- The superior radio-ulna joint, which anatomically belongs to the elbow.

- The inferior radio-ulna joint, which is anatomically separate from the wrist.

WOTAS controls the pronation-supination movement controlling the rotation state of a bar parallel to the forearm. The bar is fixed proximally over the olecranon (point B, Figure 5). Therefore it is fixed to the position of the ulna at the elbow level. The distal fixation of the bar is done over the head of the radius, although the bar is maintained in the ulnar side in order to minimize the excursion of the system. This fixation is explained later in the support design point.

2) Wrist Flexion-Extension: For orthotic purposes, flexion-extension movement is considered as a pure rotational movement with the axis of rotation in the line between the capital and lunate bones of the carpus. Therefore, for rotational actuators, the axis of rotation should be placed collinearly.

Linear actuators can be placed both in the dorsal side or in the palmar side of the forearm. A biomimetic approach to the muscle actuation lines can be achieved with this kind of actuators in order to make the orthosis more aesthetic. The most powerful muscles to control the hand and the wrist are placed close to the elbow (despite their tendons are placed over the hand). This kind of design has the advantage of reducing the volume of the hand providing the required strength to control it.

The characteristics intrinsically dynamic of tremor causes the convention orthotic systems in the market to fail when they target is tremor suppression. In these cases the tremor suppression mechanism tends to loose its alignment instead of suppressing tremor. This loose of alignment could be explained due to the support systems tends to rotate in their tip.

The attachments consist of thermoplastic material to make easy the conformation to the patient limb. This attachment provides at least three points of fixation per segment and avoids misalignments.. Velcro straps have 
been fixated to the fabric in order to tighten the support to the arm. The fixation to the wrist in mainly placed over the radial side for the reason that the wrist follows the movement of the head of the radius. Both the distal tip of the pronation-supination control and the proximal tip of the wrist control are fixed to the ulnar side of the wrist through and to the support of the wrist. The fixation to the hand is very similar to that of the wrist.

\section{B. Sensors}

The system is aimed to allowing both monitoring of tremor data and implementation of tremor suppression strategies. Therefore, it is equipped with kinematic (angular position, velocity and acceleration) and kinetic (interaction force between limb and exoskeleton) sensors.

Tremor force, position, velocity and acceleration are required in order to set up and test all different control strategies. We have restricted our analysis to the following sensors: goniometry, gyroscopes and accelerometers. The analysis of the candidate sensors was based on the Dominic method. Following this method, gyroscopes have been selected as a promising technology. The main advantages of using gyroscopes are [12]: measures rotational motion - human motion is rotational about joints, they are not influenced by gravity,both frequency \& magnitude information accurate down to DC (zero frequency), only a single integration is needed to obtain angular displacement, only a single derivation is needed to obtain angular acceleration, high signal to noise ratio, high dynamic range, solid state gyros do not influence motion of subject being measured.

Since gyroscopes provide absolute angular velocity in its active axis, the combination of two independent gyroscopes, placed distal and proximally to the joint of interest, is required. Basic electronics provide a band pass filter with a low cut-off frequency of $0.3 \mathrm{~Hz}$ and a higher cut-off frequency of $25 \mathrm{~Hz}$. This frequency band was considered the most relevant to tremor applications after a thorough literature review, [13]. The concept of using gyroscopes as an ungrounded method to assess tremor variables was evaluated and confirmed.

1) Force sensor: Since no backdrivable actuators were chosen for the application (see next section), it was also decided to use force sensors as a means of implementing impedance feedback control strategies. Strain gauges in a full Wheatstone bridge have been used as force sensors. The full Wheatstone bridge is excited with a stabilized DC supply. Additional conditioning electronics are used to balance the bridge for null point of measurement. The strain gauges should measure the torque applied by motor on WOTAS structure, so the gauges are mounted on WOTAS structure in such a way that its just measure the force perpendicular to motor shaft, their measures are not influenced by forces origin in undesired directions. The strain gauge system was characterized and the sensitivities of the system were derived in the three planes. The tests performed showed that the system has a very low sensibility for orthogonal forces. The system presented a thermal problem. As the time passes by, the temperature in the

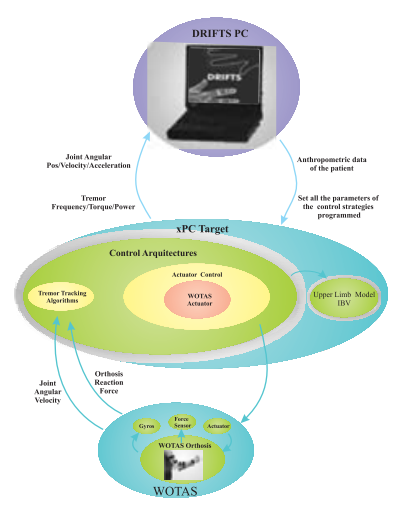

Fig. 6. WOTAS control architecture.

gauge bridge increases and this change in the temperature change the offset of the output voltage. However, after some minutes the output voltage was stable.

\section{Actuators}

Before the selection of specific actuators to suppress tremor, an estimation of the required torque and power was performed. This was achieved through the analysis of kinematics tremor data from various patients, [14]. An alternative way of obtaining torque and power is by direct measurement of tremor forces.

As in the previous section, a number of candidate actuators were evaluated. The analysis was restricted to the following actuators: Electro Active Polymers (EAPs), Electro and Magneto Rheological fluids (ERF-MRF), DC motors, Shape Memory Actuators (SMAs), Pneumatic muscles and Ultrasonic Motors.

The actuator technology should have a high power density allowing the implementation of a compact and light solution suitable for wearable devices. Based on this criteria, both DC motors and Ultrasonic motors can be regarded as the best alternatives for exerting tremor suppression forces. The former are well-known readily available technologies. They allow easy integration in advanced control schemes but they are bulky. The latter are less flexible but offer very compact solutions due to their dynamic range (low velocity high torque operating range). Both alternatives were evaluated in prototypes and DC motor eventually selected for the final version of WOTAS. The main problem with the ultrasonic motors was their poor response at low speed, therefore leading to problems to track user's slow voluntary motion, [15]. The solution selected is based on a DC motor + gear. The DC motor selected to activate WOTAS articulations is a Maxon DC flat brushless motor EC45. The gearbox used in both cases is a Harmonic Drive HDF-014-100-2A.

\section{Control Architecture}

WOTAS' control architecture is mainly composed by 3 components, namely, the exoskeleton structure with its sensors and actuators; A standard PC where the RT control algorithms of the orthosis are executed; and, A standard desktop PC that works as a host computer and implements the interface with the clinician, see Figure 6. 
This control architecture aims to interface the control algorithms with the exoskeleton. The WOTAS circuitry and sensors serve two functions: 1)Obtain the position, angular velocity and acceleration signals for control, data collection and evaluation. 2)To generate the power signal to activate the actuator.

WOTAS is able to operate basically in three control modes:

1) Monitoring mode. WOTAS operates in free mode (no force is applied to the upper limb) and monitors tremor parameters of the patients

2) Passive Control mode. WOTAS is able to change biomechanical characteristics of upper limb, such as viscosity or inertia, in order to suppress tremor. It virtually modifies the upper limb impedance.

3) Active Control mode. WOTAS is able to apply forces opposite to the tremorous movement based on a real time estimation of the involuntary component of motion. This leads to an active compensation and effective suppression of tremor.

The control of the entire active exoskeleton is being implemented in the MatLab RT suite by Mathworks. This environment provides mathematical libraries that help implement complex control strategies in real time. The interface between the MatLab environment and the active orthosis is based on a standard data acquisition board.

In order to provide an interface to all the control strategies a software application was developed in $\mathrm{C}$ language. It communicates with the low level controller (either by TPC/IP, wired serial link or BlueTooth) using Dynamic Link Libraries (DLLs), [16].

\section{BIOMECHANICAL MODEL OF THE UPPER-ARM}

A biomechanical model of the combination upper limbWOTAS has been build in order to describe its kinematics and dynamics. The biomechanical model has been build by taking into account Leva's, [17], and Zatsiorsky's, [18], tables. These tables are the most widely accepted ones in the field of biomechanics for dynamic analysis of the upper arm. Leva's adjustments have been made in order to accurately define the anthropometric measurements required to obtain inertial parameters from Zatsiorsky's tables.

A solid rigid model of the forearm has been build with the information taken from the above mentioned tables. The model has been parameterized using the DenavitHartenber approach. Finally a library has been made to allow a dynamical analysis of the system. This analysis has been done by using Fu's recursive algorithm, [19].

\section{A. Denavit-Hartenberg parametrisation}

Four rigid segments have been defined, so that all the recorded degrees of freedom can be analyzed. Each segment is responsible for a degree of freedom:

1) Elbow flexion-extension

2) Pronation-supination

3) Wrist flexion-extension

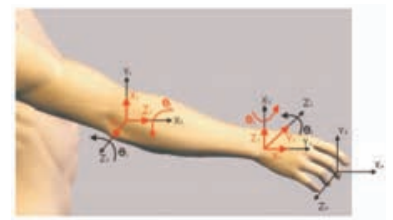

Fig. 7. Solid model representation of the forearm.

TABLE I

DH PARAMETERS

\begin{tabular}{ccccc}
\hline Segment & $\mathbf{d}$ & $\mathbf{a}$ & $\theta$ & $\alpha$ \\
\hline \hline 1 - Elbow F/E & 0 & 0 & $\beta+\pi / 2$ & $\pi / 2$ \\
2 - Pronation & $F_{L}$ & 0 & $\beta$ & $\pi / 2$ \\
3 - Wrist F/E & 0 & 0 & $\beta+\pi / 2$ & $\pi / 2$ \\
4 - Elbow Dev & 0 & $H_{L}$ & $\beta$ & $\pi / 2$
\end{tabular}

\section{4) Wrist deviation}

Two of these segments were virtual (no mass and length). In Figure 7 the various different coordinate frames and degrees of freedom are represented for each model link.

The Denavit-Hartenberg parameters can be seen in Table I. For rotary elements, the parameter $\theta$ determines the position of the joint. Therefore in the table it is indicated the relationship between the model parameter and the physiological measured angle represented by the letter $\beta$. $F_{L}$ is the length of the forearm and $H_{L}$ the length of the Hand.

\section{B. Biomechanical parameters per segment}

Biomechanical parameters per segment were obtained from Leva, [17]. Segment 1 and Segment 3 are virtual, they are only defined to manage the degrees of freedom of elbow flexion-extension and wrist Flexion-Extension respectively. All the inertial and mass parameters for each segment are defined below:

SEGMENT 1: Virtual segment SEGMENT 2:

$$
\begin{aligned}
& F_{M}=1.6 \cdot B_{M} / 100 \\
& C_{G}=\left[0, F_{L}(0.457-1), 0\right] \\
& I=\left[\begin{array}{ccc}
0.084866 & 0 & 0 \\
0 & 0.090817 & 0 \\
0 & 0 & 0.146401
\end{array}\right] \cdot F_{M} \cdot F_{L}^{2}
\end{aligned}
$$

SEGMENT 3: Virtual segment

SEGMENT 4:

$$
\begin{aligned}
& H_{M}=0.6 \cdot B_{M} / 100 \\
& C_{G}=\left[0, H_{L}(0.79-1), 0\right] \\
& I=\left[\begin{array}{ccc}
0.555185 & 0 & 0 \\
0 & 0.42397 & 0 \\
0 & 0 & 0.657553
\end{array}\right] \cdot H_{M} \cdot H_{L}^{2}
\end{aligned}
$$

where $C_{G}$ is the center of gravity, $I$ is the Inertial tensor, $B_{M}$, is the body mass, $F_{L}$, is the forearm's length, $F_{M}$ is the forearm's mass, $H_{L}$ is the hand's length and $H_{M}$, the hand's mass. 


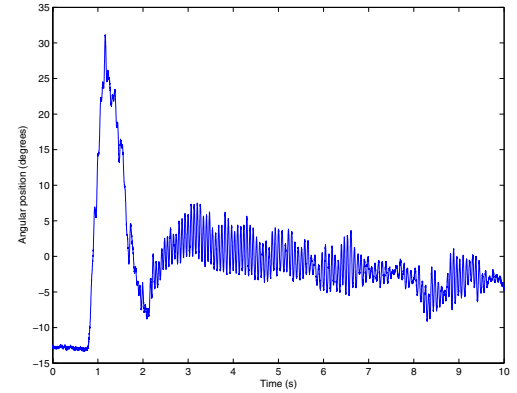

Fig. 8. Tremorous movement measured by WOTAS system. Tremor at the elbow joint in a essential tremor patient.

The biomechanical model has been implemented in Simulink in order to have a quick interface and a tool easy to integrate in the control loop of WOTAS. The computational algorithm used is based on the NewtonEuller equations of motion, see [19]. Due to its recursive implementation, these equations of motion represent an efficient set of computational equations, this allowing its implementation in real-time.

\section{Discussion}

The overall aim of this project was to developed an active robotic exoskeleton (orthosis) to provide means of testing non-grounded tremor reduction strategies in three joints of the upper limb. This paper presents a robotic orthosis platform able to monitor and control tremor in subjects. This robotic exoskeleton is equipped with kinematics (angular position, velocity and acceleration) and kinetic (interaction force between limb and orthosis) sensors. In addition, it could also apply dynamic force to the articulations of the upper limb by means of a set of flat DC motors + pancake gears.

The total weight of the final system is roughly $850 \mathrm{~g}$. A thorough programme of tests was performed to validate its wearability in daily living activities. The system was used in the laboratory to perform a wide variety of manoeuvres in free mode. These preliminary tests have successfully shown the correct operation of the system and the capability of the system to access the workspace, i.e. the system does not affect the normal range of motion of the user. The weight of the orthosis is acceptable and does not cause discomfort to the user.

The system was also evaluated with tremor patients. The patients wore WOTAS while in working and free modes. The system was able to measure and estimate tremor parameters, Figure 8. The capacity of applying dynamic internal forces to the upper limb was also evaluated and it was found that the transmission of forces from actuators to the upper limb works correctly.

The capacity of applying internal dynamic forces to the upper limb opens widely the application field of WOTAS exoskeleton. It could be applied in different areas in the field of rehabilitation robotics. It could provide restoration or maintenance of motor function to different joins of the upper limb. Most of the powered orthosis designed up to now are non-ambulatory devices, [20]. There is a clear need in the rehabilitation area of ambulatory devices able to apply dynamic forces to the upper limb.

\section{REFERENCES}

[1] J. Pons, E. Rocon, R. Ceres, D. Reynaerts, B. Saro, S. Levin, and W. V. Moorleghem, "The manus dextrous robotics upper limb prosthesis: mechanical and control aspects," Autonomous Robots, vol. 16, pp. 143-163, 2004.

[2] W. Harwin, L. Leiber, G. Austwick, and C. Dislis, "Clinical potential and design of programmable mechanical impendances for orthotic applications," Robotica, vol. 16, pp. 523-530, 1998.

[3] A. Anouti and W. Koller, "Tremor disorders: diagnosis and management," The Western Journal of Medicine, vol. 162, no. 6, pp. 523-530, 1998.

[4] R. Elble and W. Koller, Tremor. The Johns Hopkins University Press, 1990

[5] N. Hogan, "Impedance control: An approach to manipulation: Part i, ii and iii," Journal of Dynamic Systems, Measurement and Control, vol. 107, no. 1, pp. 1-23, 1985.

[6] M. Manto, M. Topping, M. Soede, J. Sanchez-Lacuesta, W. Harwin, J. Pons, J. Williams, S. Skararup, and L. Normie, "Dynamically responsive intervention for tremor suppression," IEEE Engineering in Medicine and Biology, vol. 22, no. 3, pp. 120-132, 2003.

[7] T. Krouskop, M. Krebs, I. Herskowicz, and S. Graber, "Effectiveness of mattress overlays in reducing interface pressure during recumbency," Journal of Rehabilitation Research and Development, vol. 22, pp. 7-10, 1985.

[8] R. Goonetilleke, "Designin to minimize discomfort," Ergonomics in Design, pp. 12-19, 1998.

[9] J. Gonzalez, A. García, M. Vivas, E. Ferrús, E. Alcántara, and A. Forner, A new portable method for the measurement of pressurediscomfort threshold (ptd) on the foot plant, Proceedings 4th Symposium on Footwear Biomechanics, Canmore, Canada, 1999.

[10] Guides to the evaluation of permanent impaired, American Medical Association, 1993.

[11] I. Kapandji, The physiology of the joints: Upper limb. Churchill Livingstone, 1983, vol. 1.

[12] K. Tong and M. H. Granat, "A practical gait analysis system using gyroscopes," Medical Engineering \& Physics, vol. 21, pp. 87-94, 1999.

[13] E. Rocon, J. Belda-Lois, J. Sánchez-Lacuesta, and J.L.Pons, "Pathological tremor management: Modelling,compensatory technology and evaluation," Technology and Disability, vol. 16, pp. 3-18, 2004.

[14] J. M. Belda-Lois, J. Sánchez-Lacuesta, M. Vivas-Broseta, E. Rocon, L. Bueno, and J. Pons, "Tremor movement analysis techniques: an approach towards ambulatory systems," Asistive Technology Shaping the future, pp. 827-831, 2003.

[15] E. Rocon, A. Ruiz, and J. Pons, "On the use of ultrasonic motors in orthotic rehabilitation of pathologic tremor," Proc. of ACTUATOR 2004 Conference, pp. 387-390, 2004.

[16] J. Moreno, B. Freriks, F. Thorsteinsson, J. Sánchez, and J.L.Pons, "Intelligent knee-ankle-foot orthosis: The gait project approach," Rehabilitation Sciences in the New Millenium - Challenge for Multidisciplinary Research, pp. 271-274, 2004.

[17] P. de Leva, "Adjustments to zatsioorsky-seluyanov's segment inertia parameters," Journal of Biomechanics, vol. 29, no. 9, pp. 1223-1230, 1996.

[18] V. Zatsiorsky, V. Seluyanov, and L. Chugunova, "Methods of determining mass-inertial characteristics of human body segments," in Contemporary problems of biomechanics, G. Cherny and S. Regiror, Eds. CRC Press, 1990, pp. 272-291.

[19] K. Fu, R. Gonzalez, and C. Lee, Robotics: Control, Sensing, Vision, and Intelligence. McGraw-Hill Book Company, 1987.

[20] G. Johnson, D. Carus, G. Parrini, S. S. Marchese, and R. Valeggi, "The design of a five-degree-og-freedom powered orthosis for the upper limb," vol. 25. Proceedings Instn Mech Engrs, 2002, p. H. 